\title{
Interplay between the Weibel instability and the Biermann battery in realistic laser-solid interactions
}

\author{
N. Shukla, ${ }^{1, *}$ K. Schoeffler, ${ }^{1, \dagger}$ E. Boella $\odot,{ }^{2,3}$ J. Vieira, ${ }^{1}$ R. Fonseca $\odot,{ }^{1,4}$ and L. O. Silva $\oplus^{1, \ddagger}$ \\ ${ }^{1}$ GoLP/Instituto de Plasmas e Fusão Nuclear, Instituto Superior Técnico, Universidade de Lisboa, Lisbon, Portugal \\ ${ }^{2}$ Physics Department, Lancaster University, Lancaster, England, United Kingdom \\ ${ }^{3}$ Cockcroft Institute, Sci-Tech Daresbury, Warrington, England, United Kingdom \\ ${ }^{4}$ DCTI/ISCTE, Instituto Universitario de Lisboa, Lisbon, Portugal
}

(Received 25 July 2019; accepted 8 April 2020; published 5 May 2020)

\begin{abstract}
Our setup allows the Weibel instability and its interplay with the Biermann battery to be probed in laser-driven collisionless plasmas. Ab initio particle-in-cell simulations of the interaction of short $(\leqslant 1 \mathrm{ps})$ intense $\left(a_{0} \geqslant 1\right)$ laser pulses with overdense plasma targets show observable Weibel generated magnetic fields. This field strength surpasses that of the Biermann battery, usually dominant in experiments, as long as the gradient scale length is much larger than the local electron inertial length; this is achievable by carefully setting the appropriate gradients in the front of the target, e.g., by tuning the delay between the main laser pulse and the prepulse.
\end{abstract}

DOI: 10.1103/PhysRevResearch.2.023129

The origin and evolution of magnetic fields starting from initially unmagnetized plasmas is a long-standing question, which has implications not only in astrophysics (e.g., gammaray bursts, TeV-Blazar, etc.) [1-4] but also in laboratory plasmas (e.g., fast ignition) [5-7]. Magnetic field growth in astrophysical conditions is often attributed to the turbulent dynamo mechanism, which requires an initial seed field. The dominant processes responsible for magnetogenesis, i.e., the generation of these initial fields, are still under strong debate. Among the known mechanisms, the Biermann battery and the Weibel or current filamentation instability are two major candidates [8-14]. The Biermann battery acts in the presence of temperature and density gradients perpendicular to each other $[15,16]$. In contrast, the Weibel instability is driven by temperature anisotropies $[17,18]$. These two mechanisms have been reproduced using independent scaled experiments governed by similar physical laws $[19,20]$. However, the interplay between the Biermann battery effect and the Weibel instability has not been studied in the laboratory or astrophysical contexts, making it both of fundamental interest and relevant to understand magnetogenesis. In this paper, we propose an experimental setup to explore the interplay between the two plasma processes.

Recent developments in laser technology (intensities in excess of $10^{19} \mathrm{~W} / \mathrm{cm}^{2}$ with laser pulse durations shorter than $1 \mathrm{ps}$ and high-resolution diagnostics) open the possibility to

\footnotetext{
*nshukla@ist.utl.pt

${ }^{\dagger}$ kevin.schoeffler@tecnico.ulisboa.pt

‡luis.silva@tecnico.ulisboa.pt
}

Published by the American Physical Society under the terms of the Creative Commons Attribution 4.0 International license. Further distribution of this work must maintain attribution to the author(s) and the published article's title, journal citation, and DOI. probe such processes through laser-solid interactions [19,2124]. In these experiments, the magnetic field generation is often attributed to the Biermann battery $[20,25,26]$. The Biermann field grows linearly as $B(t) \approx-\left(t c / n_{e} e\right) \nabla n_{e} \times \nabla T_{e} \approx$ $(t c / e)\left(k_{B} T_{e} / L_{T} L_{n}\right)$, where $L_{n} \equiv n_{e} / \nabla n_{e}$ and $L_{T} \equiv T_{e} / \nabla T_{e}$ are the density and temperature gradient scale lengths, respectively; $k_{B}$ is the Boltzmann constant; $n_{e}$ and $T_{e}$ are the electron density and temperature; $e$ is the elementary charge; and $c$ is the speed of light in vacuum. Theoretical and computational studies have demonstrated magnetic field generation via the Biermann battery $[27,28]$ in the context of hydrodynamical systems. Recently, Schoeffler et al. [29,30] investigated the kinetic effects of the Biermann battery in a collisionless expanding plasma, finding that for sufficiently large gradient scale length $L \sim L_{n} \sim L_{T}$ the Weibel instability competes with the Biermann battery. The relative importance of the Biermann battery can be adjusted by changing the scale length of the density and temperature gradients. For systems where $L / d_{e}<100$, the saturated Biermann battery generated field obeys the scaling

$$
\frac{B}{\sqrt{8 \pi P_{\text {plasma }}}}=\beta_{e}^{-1 / 2} \sim \frac{d_{e}}{L},
$$

where $P_{\text {plasma }}$ is the plasma pressure, $d_{e} \equiv c / \omega_{p}$ and $\omega_{p}=$ $\left(4 \pi e^{2} n_{e} / m_{e}\right)^{1 / 2}$ are the respective electron skin depth and plasma frequency, and $m_{e}$ is the electron rest mass. The saturation of the Weibel instability is independent of the system size, occurring when plasma $\beta \sim 1$. The intersection between the numerically determined saturation of the Biermann and Weibel fields occurs at $L / d_{e}=100[29,30]$, and therefore when $L / d_{e} \geqslant 100$ the Weibel instability generates magnetic fields that overpower that of the Biermann battery.

In this paper, we carry out a numerical and theoretical study using particle-in-cell (PIC) simulations to investigate magnetic fields generated by the Weibel instability in the 
interaction of a short $(\leqslant 1 \mathrm{ps})$ high intensity $\left(a_{0} \geqslant 1\right)$ laser pulse and a plasma with sufficiently large $L$. Until now, the large simulation domains and long simulation times required to capture these mechanisms have impeded detailed exploration of this regime. Our simulation results reveal that by tuning the delay between an ionizing prepulse and the main pulse, and defining the spot size of the laser such that $L / d_{e} \geqslant$ 100 , the Weibel generated magnetic field magnitude surpasses the usually observed Biermann field, and can be directly observed in current laser-plasma interaction experiments.

We simulate the interaction of an ultraintense laser pulse with a fully ionized unmagnetized electron-proton plasma with realistic mass ratio (proton mass $m_{i}=1836 m_{e}$ ) using the OSIRIS framework [31-33]. The laser is $s$ polarized (i.e., the electric field is perpendicular to the simulation plane) and has a peak intensity $I_{L}=10^{19} \mathrm{~W} / \mathrm{cm}^{2}$ (normalized vector potential $a_{0}=2$ ) with a wavelength $\lambda_{0}=1.0 \mu \mathrm{m}$. We choose $s$ polarization to isolate the out-of-plane Biermann and Weibel magnetic fields from the laser field. We performed smaller two- and three-dimensional simulations, finding that $s$ polarization in two dimensions better approximates threedimensional conditions, as both conditions have been shown to produce less heating than with $p$ polarization in two dimensions (see Refs. [28,34]). We define $\omega_{p}$ and $d_{e}$ using a reference plasma density $n_{0}=1.1 \times 10^{22} \mathrm{~cm}^{-3}=10 n_{c}$, where $n_{c}=\omega_{0}^{2} m_{e} / 4 \pi e^{2}$ is the critical density, and $\omega_{0}=2 \pi c / \lambda_{0}$ is the laser frequency. The envelope of the pulse follows a flat-top function having rise $(R)$ and fall $(F)$ time $\tau_{R}=$ $\tau_{F}=10.0 \omega_{p}^{-1}(1.7 \mathrm{fs})$ and duration $\tau_{F T}=1034 \omega_{p}^{-1}(175 \mathrm{fs})$. Its transverse profile is modeled as a Gaussian function with spot size at full width at half maximum $(\mathrm{FWHM}) \mathrm{w}_{\mathrm{FWHM}}=$ $100 d_{e}(5 \mu \mathrm{m})$. These are typical laser parameters in lasersolid interaction experiments [35].

The laser (propagating along the $x_{1}$ direction) interacts with a plasma having longitudinal electron density profile $n_{e}\left(x_{1}\right)=0.5 n_{0}\left\{\tanh \left[2\left(x_{1}-x_{10}\right) / L_{n}\right]+1\right\}$, where $n_{0}=10 n_{c}$ is the maximum density, $x_{1}$ is the longitudinal coordinate, and $L_{n}\left[=n_{0} / \nabla n_{e}\left(x_{10}\right)\right]$ is the initial density scale length where in our primary simulation $L_{n}=400 d_{e}(20 \mu \mathrm{m})$. The laser focal point coincides with the location of critical density at $x_{10}=$ $1250 d_{e}$. The electrons and ions have initial temperatures $T_{e 0}=1 \mathrm{keV}$ and $T_{i 0}=1 \mathrm{eV}$, respectively (small compared to the laser heating, but large enough to resolve the Debye length).

The simulation box size $L_{x 1} \times L_{x 2}=2000 \times 2000 d_{e}^{2}$ is divided into $20000 \times 20000$ cells and a time step $\Delta t=$ $0.05 \omega_{p}^{-1}$. Each cell contains 12 macroparticles per species, the dynamics of which have been followed for more than 100000 time steps. We choose absorbing boundary conditions along $x_{1}$ and periodic conditions along $x_{2}$ for fields and particles. Increased transverse box sizes $L_{x 2}$, spatial and temporal resolution, and number of particles per cell were tested, showing overall convergence.

We focus our observations on the magnetic field at the front surface of the target, choosing the length of the target long enough that the back side does not influence the front (we have checked that the particles reflecting from the back do not reach the region $x_{1}<1150 d_{e}$ where significant heating occurs until after $\left.t=2812.60 \omega_{p}^{-1}\right)$, and $n_{e}=0$ at the right wall to avoid significant particle loss at the boundary. We

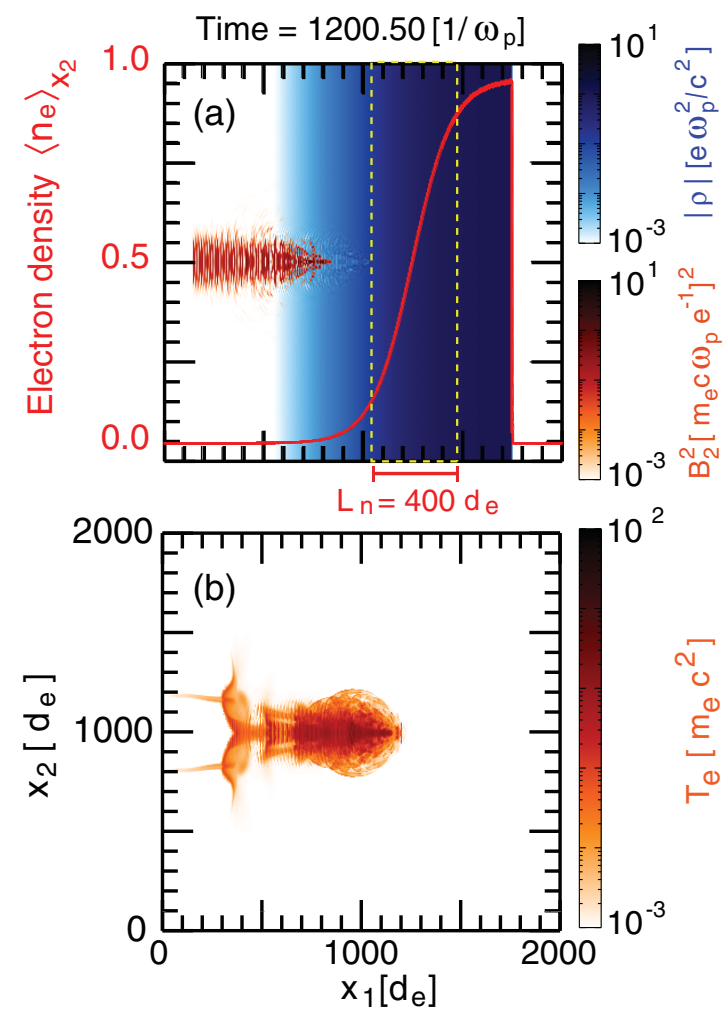

FIG. 1. Electron density $n_{e}$ (blue) and laser magnetic field $B_{2}^{2}$ (orange) (a) and electron temperature $T_{e}$ (b) at $t=1200.50 \omega_{p}^{-1}$. The red solid line in (a) is an average of the density along the $x_{2}$ direction, and the dashed red line shows the gradient length scale $L_{n}=400 d_{e}$.

choose a step function at $x_{1}=1750 d_{e}$ to minimize the length and save computational time [see Fig. 1(a)].

Figure 1 shows, in the simulation where $L_{n}=400 d_{e}$, that the laser produces temperature gradients that are not aligned with the density gradient associated with $L_{n}$. The laser enters the simulation domain from the left and at time $t \simeq 1200.50 \omega_{p}^{-1}$ penetrates the plasma up to $1000 d_{e}$ [Fig. 1(a)]. The interaction of the laser with the plasma resonantly heats the electrons, consistent with the scaling of Ref. [36] [Fig. 1(b)]. The temperature is defined as $T_{e}=$ $\operatorname{Tr}\left(T_{i j}\right) / 3$, where $T_{i j} \equiv \int\left(u_{i} u_{j} / \gamma\right) f(u) d^{3} u / \int f(u) d^{3} u$, calculated in the rest frame, is the temperature tensor; $u_{i}$ is the normalized proper velocity; $\gamma=\sqrt{1+u^{2}}$; and $f(u)$ is the velocity distribution function. By time $t \simeq 2812.60 \omega_{p}^{-1}$, the laser has created a conical shaped channel [see Fig. 2(a)] and induced a large thermal gradient with $L_{T}=1000 d_{e}$ pointing radially towards the axis of the laser beam [see Fig. 2(b)]. The temperature gradient is not aligned with the density gradient along $x_{1}$, allowing the Biermann battery to generate a toroidal B field.

The average temperature along the line at $x_{1}=700 d_{e}$ is $\left\langle T_{e}\right\rangle_{x 2}=0.34 m_{e} c^{2}$ (see Fig. 1). Given this temperature and the maximum density $n_{0}=1.1 \times 10^{22} \mathrm{~cm}^{-3}$, we conservatively estimate the collisionality. The ratio of $L_{n}$ to the electron collisional mean free path $l_{e}[37] L_{n} / l_{e}=0.00047 \ll$ 1 , therefore we neglect collisions.

Figure 3 shows the Bierman-produced out-of-plane magnetic field $B_{3}$ at $t=2641.10 \omega_{p}^{-1}$ in the region $x_{1}<700 d_{e}$. 


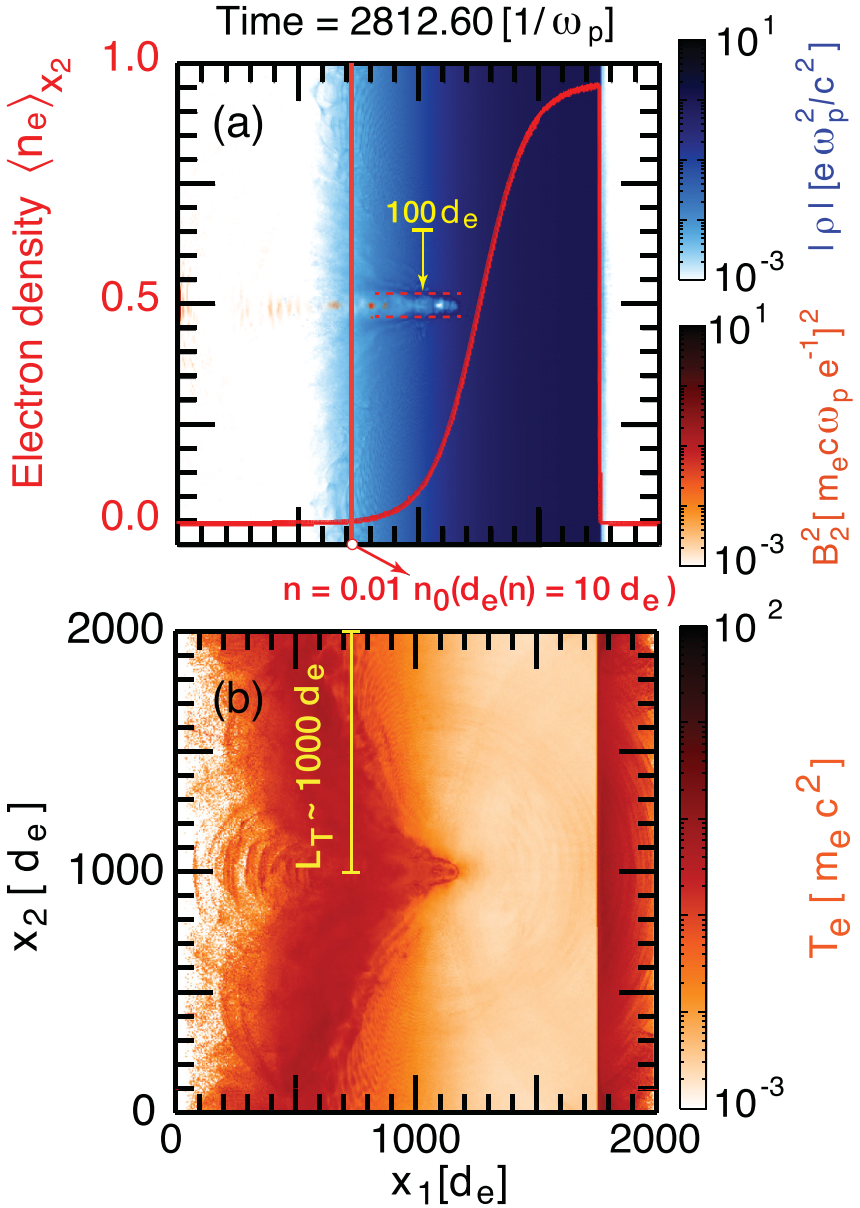

FIG. 2. Electron density $n_{e}$ (blue) and laser magnetic field $B_{2}^{2}$ (orange) (a) and electron temperature $T_{e}$ (b) at $t=2812.6 \omega_{p}^{-1}$. The red dashed box in (a) indicates the focal spot of the laser. The red dashed line defines the boundary between $L_{n}>100 d_{e}\left(n_{e}\right)$ (left) and $L_{n}<100 d_{e}\left(n_{e}\right)$ (right). The blue dashed lines in (b) point to the location where $L_{T} \simeq 1000 d_{e}$.

However, alongside the Biermann-generated field, in the region $x_{1}>700 d_{e}$, a field due to the Weibel instability is also observed. The magnetic field reaches a maximum amplitude of the order of $0.065 m_{e} c / e \omega_{p}(22 \mathrm{MG})$. Note that a lowpass filter was applied to the magnetic field only allowing wavelengths above $31.4 d_{e}(1.57 \mu \mathrm{m})$, mimicking the typical experimental resolution (see, e.g., Ref. [35]). The boundary between Biermann and Weibel regimes is estimated at the location where $L_{T}\left(x_{1}\right) / d_{e}\left[n_{e}\left(x_{1}\right)\right] \approx 100$ [29,30], where $d_{e}\left[n_{e}\left(x_{1}\right)\right]$ is the local electron inertial length. Remarkably, this transition occurs precisely at $x_{1}=700 d_{e}$, indicated by the dotted vertical line in Fig. 3(a), as $d_{e}\left[n_{e}\left(x_{1}\right)\right]=10 d_{e}$ and $L_{T}\left(x_{1}\right)=1000 d_{e}$ [see Figs. 2(a) and 2(b)].

Figure 4(a) shows the temporal evolution of the square root of the average out-of-plane magnetic energy density $\left\langle B_{3}^{2}\right\rangle^{1 / 2}$ in the region $x_{1}=[800-900] d_{e}, x_{2}=[600-900] d_{e}$, where the dominant source of the magnetic field is the Weibel instability. Between 2000 and $3000 \omega_{p}^{-1}$, after the laser has passed this region [see Fig. 4(b)], the laser magnetic fields are no longer present. Here, we observe an exponential growth of the magnetic field $\left(\Gamma_{\text {sim }}=0.0015 \omega_{p}\right.$ with a corresponding wave

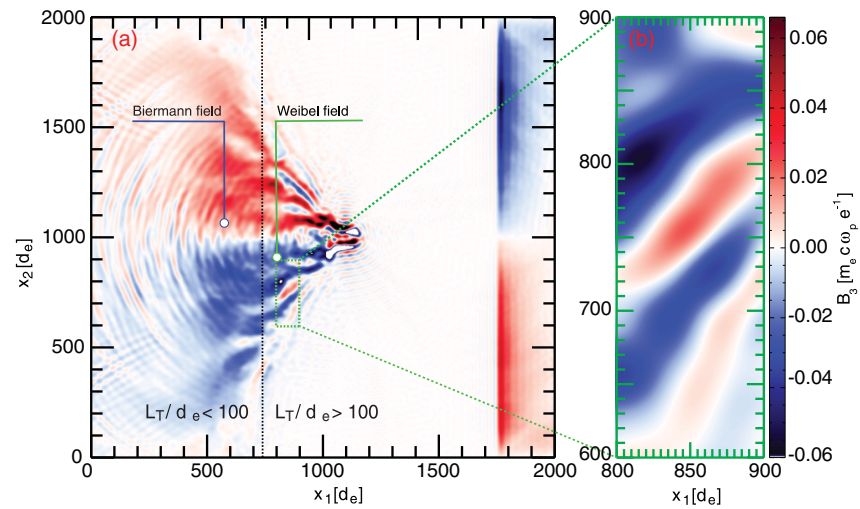

FIG. 3. (a) Out-of-plane magnetic field $B_{3}$ at $t=$ $2641.10 \omega_{p}^{-1}(440 \mathrm{fs})$ and (b) zoom-in of Weibel-generated magnetic filaments with $k \approx 0.06 d_{e}^{-1}$. The black dashed line in (a) indicates the transition point between the region where Biermann fields dominate $\left(L_{T} / d_{e}<100\right)$ and the region where Weibel fields dominate $\left(L_{T} / d_{e}>100\right)$.

vector $k \simeq 0.15 d_{e}^{-1}$, agreeing reasonably with theory from Ref. [38]). The spatiotemporal evolution of the laser magnetic field energy shown in Fig. 3(b) shows that the end of the laser pulse passes the region where we calculate the growth rate $\left(x_{1}<900 d_{e}\right)$ at $t=1950 \omega_{p}^{-1}(322 \mathrm{fs})$. Meanwhile, the expansion of the hot energetic electron population generated via laser heating contributes to the average anisotropy in the velocity distribution [see Fig. 4(c)] [39]. The anisotropy $A \equiv$ $T_{\text {hot }} / T_{\text {cold }}-1$, where $T_{\text {hot }}$ and $T_{\text {cold }}$ are the respective larger and smaller eigenvalues of the temperature tensor $T_{i j}$, provides the free energy that drives the Weibel instability.
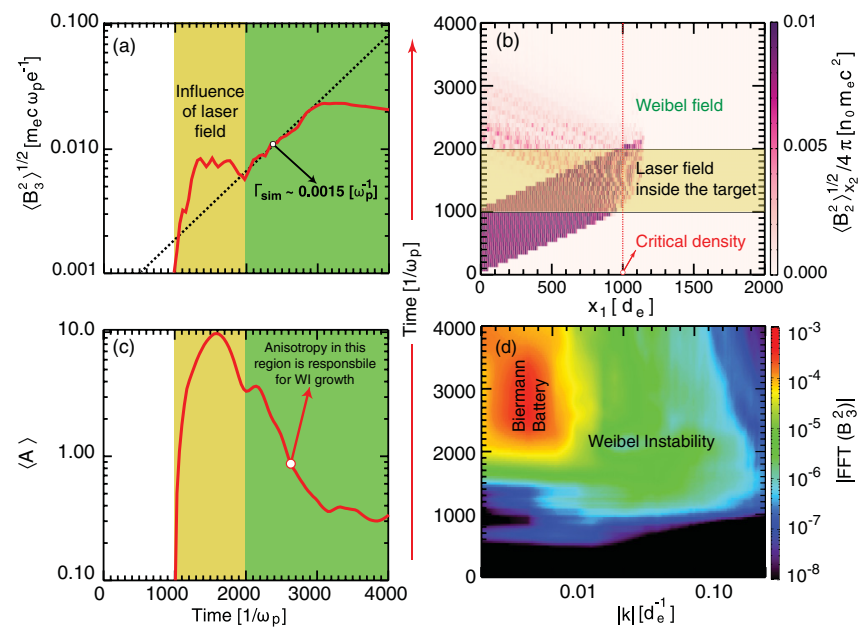

FIG. 4. (a) Temporal evolution of the square root of the average out-of-plane magnetic energy density $\left\langle B_{3}^{2}\right\rangle^{1 / 2}$ in the green box indicated in Fig. 3. The slope of the curve in (a), identified as the Weibel growth rate, is $\simeq 0.0015 \omega_{p}$ (black dashed line). The temporal evolution of the magnetic field energy associated with the laser $\left\langle B_{2}^{2}\right\rangle_{x_{2}}$, averaged along $x_{2}$, as a function of $x_{1}$ is plotted in (b). The average anisotropy $\langle A\rangle$ in the green box indicated in Fig. 3 is shown in (c). The temporal evolution of the transverse magnetic field energy $B_{3}^{2}$ spectrum in (d) shows the contribution to the B field from the Weibel instability and the Biermann battery. 


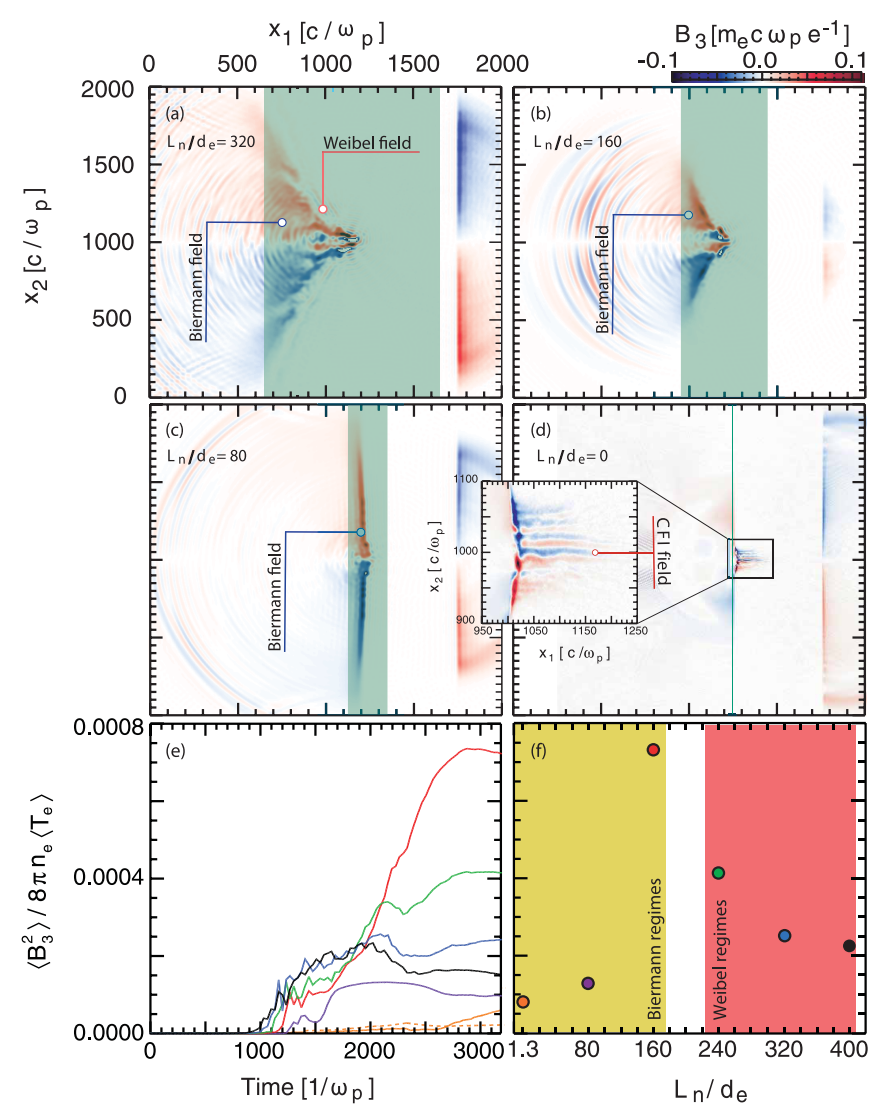

FIG. 5. Out-of-plane magnetic field $B_{3}$ for $L_{n} / d_{e}=320$ (a), 160 (b), 80 (c) (with low-pass filter), and 0 (d) (without low-pass filter) at time $t=2023.70 \omega_{p}^{-1}$. Shaded regions indicate where the meanfield energy was averaged between $x_{1}=1250 d_{e}-1.875 L_{n}$ and $1250 d_{e}+1.25 L_{n}$. A zoom of the region where the current filamentation is found is included in (d). The temporal evolution of the average out-of-plane magnetic energy density $\left\langle B_{3}^{2}\right\rangle$ [averaged over the specified regions highlighted in (a)-(d) with low-pass filter] is shown in (e). The dashed line shows the average magnetic energy density (without low-pass filter) in the range $x_{1}=[950-1250] d_{e}$. The peak $\left\langle B_{3}^{2}\right\rangle$ is plotted as a function of $L_{n} / d_{e}$ in (f). The Biermann field dominates over the Weibel field where $L_{n} / d_{e} \geqslant 160$ (yellow region), while a region where the Weibel field dominates exists when $240 \geqslant L_{n} / d_{e}$ (red region).

The time varying spectrum of $B_{3}^{2}$ in Fig. 4(d) shows the contribution of the Weibel instability and the Biermann battery to the magnetic field energy. The spectra are obtained by performing a Fourier transform over the entire system for the out-of-plane magnetic fields, and then averaging over all directions of $\mathbf{k}$. With the $\log$ scale it is not obvious that the energy contained in the Weibel magnetic fields is comparable to that of the Biermann field. The Biermann magnetic field energy $\left(k d_{e}<0.025\right)$ remains about five times higher than the Weibel magnetic field energy $\left(k d_{e}>0.025\right)$ after $t=$ $2370 \omega_{p}^{-1}$.

We performed a parameter scan for $L_{n} / d_{e}=0,80$, $160,240,320$, and 400 . Note that by the time the laser reaches the target at $t \sim 1250 \omega_{p}^{-1}$ the length scale rises by $\sim \sqrt{k_{B} T_{e 0} / m_{i}} t \sim 1.3 d_{e}$, given $T_{e 0}=1 \mathrm{keV}$. Therefore, for $L_{n} / d_{e}=0$, the effective density scale length is $1.3 d_{e}$.
Figures 5(a)-5(d) show $B_{3}$ at time $t=2023.70 \omega_{p}^{-1}$ [when the Weibel generated magnetic fields saturate in the $L_{n} / d_{e}=400$ case; see Fig. 5(e)] for a selection of $L_{n} / d_{e}$. With a target of sufficiently large $L_{n} / d_{e}>160$, a region of Weibel generated magnetic fields is visible [see Fig. 5(a) where $L_{n} / d_{e}=320$ ]. However, for $L_{n} / d_{e} \leqslant 160$, the Biermann magnetic field dominates, and no region exists where the Weibel instability is prominent [see Figs. 5(b)-5(d)].

Thin filaments in $B_{3}$ explained by the current filamentation instability (CFI) $[7,40,41]$ are observed in many experiments $[42,43]$ where a laser hits a plasma target with a sharp density profile. Figure 5(d) shows these filaments (without the low-pass filter). Unlike the Weibel generated field described in this paper, a sharp relativistic electron beam provides the free energy rather than the thermal expansion of the plasma. In our simulations, the CFI field is much weaker than both the Weibel and Biermann fields for other $L_{n} / d_{e}$. Furthermore, in this paper, we focus on the region with density and temperature gradients that lead to the Biermann battery and Weibel instability, rather than deep inside the target where these thin filamentary fields are found.

The magnetic energy density produced from the laser interaction depends on $L_{n}$. Figure 5(e) shows the temporal evolution of the average out-of-plane magnetic energy density $\left\langle B_{3}^{2}\right\rangle$ (with low-pass filter) in the region between $x_{1}=$ $1250 d_{e}-1.875 L_{n}$ and $1250 d_{e}+1.25 L_{n}$ for each simulation [see highlighted regions in Figs. 5(a)-5(d)]. Weibel fields are observed when $L_{n} / d_{e}>160$, saturating at $t \sim 2000 \omega_{p}^{-1}$. For all cases, the Biermann field grows and saturates after $t \geqslant 2150 \omega_{p}^{-1}$. The dashed line shows $\left\langle B_{3}^{2}\right\rangle$ (without low-pass filter) in the range $x_{1}=[950-1250] d_{e}$ associated with the zoomed region in Fig. 5(d), which peaks at $t \sim 2000 \omega_{p}^{-1}$. This CFI magnetic field is much smaller than the dominant fields for bigger $L_{n}$. In Fig. 5(f), the peak $\left\langle B_{3}^{2}\right\rangle$ is shown as a function of $L_{n} / d_{e}$. The maximum $\left\langle B_{3}^{2}\right\rangle$ occurs at $L_{n} / d_{e}=160$, the transition between the Biermann and Weibel regimes.

The transition between the regimes where only the Biermann battery is present $\left(L_{n} / d_{e}<160\right)$ and both the Weibel instability and the Biermann battery are present $\left(L_{n} / d_{e}>\right.$ 160) can be probed experimentally. After the target is ionized by the prepulse, the plasma expands, resulting in a nonuniform density with a gradient length that can reach several micrometers when the main pulse arrives. A possible model for the density scale length as a function of time yields $L_{n}(t)=14.5 \mu \mathrm{m} \times \bar{I}_{L}^{10 / 27} \overline{\mathrm{A}}^{-2 / 27} \bar{\Lambda}^{4 / 27} \bar{\lambda}_{L}^{4 / 9} \overline{\Delta t}{ }^{31 / 27}$ [44], where the bar notation signifies quantities normalized to a typical prepulse laser with intensity of $I_{L}=10^{12} \mathrm{~W} / \mathrm{cm}^{-2}$, the nuclear mass number $A=2$, the Coulomb logarithm $\Lambda=5$, the laser wavelength $\lambda_{0}=1 \mu \mathrm{m}$, and pulse delay $\Delta t=200 \mathrm{ps}$. For example, with these scalings, pulse delays 278 and 68.4 ps correspond with $L_{n}=400 d_{e}$ and $80 d_{e}$, confirming the experimental feasibility of these density scale lengths.

Magnetic fields can be measured using the synchrotron radiation in addition to the conventional method of proton radiography [45]. For the parameters of this paper, radiation will have wavelength estimated between 190 and $1200 \mathrm{~nm}$, while for higher-power lasers this signal would become stronger 
and approach x-ray frequencies. The detailed prediction of the radiation spectra, which can in principle be performed using radiation algorithms [46,47], will be left for future work.

In this paper, we have demonstrated the possibility to clearly observe the generation of electron Weibel magnetic fields in laboratory experiments. First-principles PIC simulations of the interaction of an intense laser pulse with an overdense plasma target have demonstrated the Weibel instability in the presence of sufficiently weak gradients at the front of the target $\left(L_{n} / d_{e} \geqslant 160\right.$ and $\left.\mathrm{w}_{\mathrm{FWHM}}=100 d_{e}\right)$. The Weibel instability is driven by an electron pressure anisotropy caused by the rapid expansion of the electrons in the front of the target, following the laser-plasma interaction. The Weibel instability produces fields saturating at magnitudes comparable to the Biermann fields.
Finally, we note that density gradients needed to observe the instability at work could easily be achieved tuning the delay between the ionizing prepulse and the main pulse at existing laser facilities. For instance, facilities such as the Vulcan laser facility at Rutherford Appleton Laboratory [48] with a peak intensity around $I_{L}=10^{19} \mathrm{~W} / \mathrm{cm}^{2}$, wavelength $\lambda_{0}=1.054 \mu \mathrm{m}$, a duration of hundreds of femtoseconds, and a contrast of $10^{7}$ would easily allow testing the interplay and the competition between the Weibel and the Biermann mechanisms.

This work was partially supported by the European Research Council (Grant No. ERC-2015-InPairs-695088). Simulations were performed at the IST cluster (Lisbon, Portugal) and on the Marconi supercomputer (CINECA) in the framework of the HPC-Europa3 program.
[1] D. A. Uzdensky and S. Rightley, Rep. Prog. Phys. 77, 036902 (2014).

[2] L. W. Widrow, Rev. Mod. Phys. 74, 775 (2002).

[3] P. P. Kronberg, Phys. Today 55, 40 (2002).

[4] R. M. Kulsrud and E. G. Zweibel, Rep. Prog. Phys. 71, 046901 (2008).

[5] L. O. Silva, R. A. Fonseca, J. W. Tonge, W. B. Mori, and J. M. Dawson, Phys. Plasmas 9, 2458 (2002).

[6] M. Tzoufras, C. Ren, F. S. Tsung, J. W. Tonge, W. B. Mori, M. Fiore, R. A. Fonseca, and L. O. Silva, Phys. Rev. Lett. 96, 105002 (2006).

[7] N. Shukla, A. Stockem, F. Fiuza, and L. O. Silva, J. Plasma Phys. 78, 181 (2012).

[8] M. V. Medvedev and A. Leob, Astrophys. J. 526, 697 (1999).

[9] R. A. Fonseca, L. O. Silva, J. Tonge, R. G. Hemker, J. M. Dawson, and W. B. Mori, IEEE Trans. Plasma Sci. 30, 28 (2002).

[10] M. V. Medvedev, M. Fiore, R. A. Fonseca, L. O. Silva, and W. B. Mori, Astrophys. J. 618, L75 (2004).

[11] K. I. Nishikawa, J. Niemiec, P. E. Hardee, M. Medvedev, H. Sol, Y. Mizuno, B. Zhang, M. Pohl, M. Oka, and D. H. Hartmann, Astrophys. J. Lett. 698, L10 (2009).

[12] N. Shukla and P. K. Shukla, J. Plasma Phys. 76, 1 (2010).

[13] A. Bret, Astrophys. J. 699, 990 (2009).

[14] C. Ruyer, L. Gremillet, G. Bonnaud, and C. Riconda, Phys. Rev. Lett. 117, 065001 (2016).

[15] A. Gruzinov, Astrophys. J. Lett. 563, L15 (2001).

[16] A. Brandenburg, D. Sokoloff, and K. Subramanian, Space Sci. Rev. 169, 123 (2012).

[17] E. S. Weibel, Phys. Rev. Lett. 2, 83 (1959).

[18] A. Bret, L. Gremillet, and M. E. Dieckmann, Phys. Plasmas 17, 120501 (2010).

[19] M. Tatarakis, I. Watts, F. N. Beg, E. L. Clark, A. E. Dangor, A. Gopal, M. G. Haines, P. A. Norreys, U. Wagner, M.-S. Wei et al., Nature (London) 415, 280 (2002).

[20] S. Mondal, V. Narayanan, W. J. Ding, A. D. Lad, B. Hao, S. Ahmad, W. M. Wang, Z. M. Sheng, S. Sengupta, P. Kaw et al., Proc. Natl. Acad. Sci. USA 109, 8011 (2012).

[21] C. Ruyer, L. Gremillet, and G. Bonnaud, Phys. Plasmas 22, 082107 (2015).
[22] F. Fiuza, R. A. Fonseca, J. Tonge, W. B. Mori, and L. O. Silva, Phys. Rev. Lett. 108, 235004 (2012).

[23] E. Boella, F. Fiúza, A. S. Novo, R. A. Fonseca, and L. O. Silva, Plasma Phys. C. F. 60, 035010 (2018).

[24] N. Shukla, P. K. Shukla, and L. Stenflo, Phys. Rev. E 80, 027401 (2009).

[25] J. A. Stamper, K. Papadopoulos, R. N. Sudan, S. O. Dean, E. A. McLean, and J. M. Dawson, Phys. Rev. Lett. 26, 1012 (1971).

[26] Y. Sakagami, H. Kawakami, S. Nagao, and C. Yamanaka, Phys. Rev. Lett. 42, 839 (1979).

[27] M. G. Cadjan, M. F. Ivanov, and A. V. Ivlev, Laser Par. Beams 15, 33 (1997).

[28] S. C. Wilks and W. L. Kruer, IEEE J. Quantum Elect. 33, 1954 (1997).

[29] K. M. Schoeffler, N. F. Loureiro, R. A. Fonseca, and L. O. Silva, Phys. Rev. Lett. 112, 175001 (2014).

[30] K. M. Schoeffler, N. F. Loureiro, R. A. Fonseca, and L. O. Silva, Phys. Plasmas 23, 056304 (2016).

[31] R. A. Fonseca, L. O. Silva, F. S. Tsung, Decyk, V. K., W. Lu, C. Ren, W. B. Mori, S. Deng, S. Lee et al., Lect. Notes Comput. Sci. 2331, 046401 (2002).

[32] R. A. Fonseca, S. F. Martins, L. O. Silva, J. W. Tonge, F. S. Tsung, and W. B. Mori, Plasma Phys. C F 50, 124034 (2008).

[33] R. A. Fonseca, J. Vieira, F. Fiuza, A. Davidson, F. S. Tsung, W. B. Mori, and L. O. Silva, Plasma Phys. C F 55, 124011 (2013).

[34] L. Chopineau, A. Leblanc, G. Blaclard, A. Denoeud, M. Thvenet, J.-L. Vay, G. Bonnaud, P. Martin, H. Vincenti, and F. Quéré, arXiv:1707.06390.

[35] M. S. Wei, F. N. Beg, E. L. Clark, A. E. Dangor, R. G. Evans, A. Gopal, K. W. D. Ledingham, P. McKenna, P. A. Norreys, M. Tatarakis et al., Phys. Rev. E 70, 056412 (2004).

[36] A. Pukhov, Z.-M. Sheng, and J. M. ter Vehn, Phys. Plasmas 6, 2847 (1999).

[37] J. D. Huba, NRL Plasma Formulary (Naval Research Laboratory, Washington, DC, 2013).

[38] H. H. Kaang, C.-M. Ryu, and P. H. Yoon, Phys. Plasmas 16, 082103 (2009).

[39] K. M. Schoeffler, N. F. Loureiro, and L. O. Silva, Phys. Rev. E 97, 033204 (2018). 
[40] B. D. Fried, Phys. Fluids 2, 337 (1959).

[41] P. Kolodner and E. Yablonovitch, Phys. Rev. Lett. 43, 1402 (1979).

[42] M. Tatarakis, A. Gopal, I. Watts, F. N. Beg, A. E. Dangor, K. Krushelnick, U. Wagner, P. A. Norreys, E. L. Clark, M. Zepf $e t$ al., Phys. Plasmas 9, 2244 (2002).

[43] S. Göde, C. Rödel, K. Zeil, R. Mishra, M. Gauthier, F.-E. Brack, T. Kluge, M. J. MacDonald, J. Metzkes, L. Obst et al., Phys. Rev. Lett. 118, 194801 (2017).

[44] Y. J. Li and J. Zhang, Phys. Rev. E 63, 036410 (2001).
[45] C. K. Li, F. H. Séguin, J. A. Frenje, J. R. Rygg, R. D. Petrasso, R. P. J. Town, O. L. Landen, J. P. Knauer, and V. A. Smalyuk, Phys. Rev. Lett. 99, 055001 (2007).

[46] J. L. Martins, S. F. Martins, R. A. Fonseca, and L. O. Silva, Proc. SPIE 7359, 73590V (2009).

[47] M. Pardal, A. Sainte-Marie, A. Reboul-Salze, J. Vieira, and R. A. Fonseca, in Proceedings of the 45th EPS Conference on Plasma Physics, 2018 (unpublished).

[48] www.clf.stfc.ac.uk/Pages/Vulcan-laser.aspx. 